\title{
EDITORIAL
}

\section{SOBRE LA SALUD PÚBLICA BASADA EN PRUEBAS}

\author{
Jesús López Alcalde $(1,2)$ y Xavier Bonfill $(2,3,4)$
}

(1) Servicio de Medicina Preventiva y Salud Pública. Hospital General Universitario de Guadalajara.

(2) Servicio de Epidemiología Clínica y Salud Pública. Centro Cochrane Iberoamericano. Hospital de la Santa Creu i Sant Pau. Barcelona.

(3) Universitat Autònoma de Barcelona.

(4) CIBER de Epidemiología y Salud Pública (CIBERESP).

\section{LA MEDICINA BASADA EN LA EVIDENCIA Y LA SALUD PÚBLICA}

Gordon Guyatt, en un editorial de la revista ACP Journal Club publicado en el año $1991^{1}$, por primera vez introdujo en la literatura médica el término de Medicina Basada en la Evidencia (MBE), que consiste en basar la toma de decisiones clínicas en las mejores pruebas procedentes de la investigación publicadas en la literatura médica. Progresivamente, la MBE se ha consolidado como una metodología imprescindible dentro de la Medicina pero, a su vez, su concepción inicial exclusivamente clínica se ha ido ampliando para incluir también la aportación de otras disciplinas ${ }^{2}$. La definición de "Atención Sanitaria Basada en la Evidencia" (Evidence-based Healthcare $)^{3}$ por Muir Gray ya fue pionera en este sentido. No obstante, es destacable que una metodología similar todavía no se haya consolidado en el ámbito de la salud pública.

El concepto de salud pública se ha definido desde múltiples y variadas perspectivas. Entre las definiciones propuestas podemos citar la ya clásica definición de Acheson $^{4}$, que describe la salud pública como "la ciencia y el arte de prevenir la enfermedad, prolongar la vida y promover la salud mediante los esfuerzos organizados de la sociedad". Por otra parte, Frenk ${ }^{5}$ considera que la salud pública consiste en la aplicación de un conjunto de disciplinas académicas, como pueden ser las ciencias biológicas, sociales y del comportamiento, al estudio de los fenómenos relacionados con la salud de las poblaciones humanas. Sin embargo, la mayoría de las definiciones suelen presentar elementos comunes como, por ejemplo, su amplio ámbito de actuación, su base multidisciplinar y su impacto multisectorial $^{6}$.

\section{NECESIDAD DE EVALUAR RIGUROSAMENTE LAS INTERVENCIONES EN SALUD PÚBLICA}

Actualmente existe un creciente interés por conocer los resultados de las intervenciones en salud pública ${ }^{7}$ motivado, entre otros factores, por la necesidad de priorizar la inversión en intervenciones de eficacia ya demostrada. No obstante, el contexto de planificación e implementación de este tipo de intervenciones tiende a dificultar su adecuada evaluación. Entre las barreras existentes destacan la insuficiente tradición y formación en metodología evaluativa entre los profesionales de la salud pública, la 
habitual evaluación de los resultados por los propios responsables del diseño y/o aplicación de la intervención (con la consecuente pérdida de objetividad), y la frecuente necesidad de dar una respuesta rápida a las preguntas planteadas ${ }^{7}$.

Por otra parte, las intervenciones en salud pública presentan peculiaridades que las diferencian de las intervenciones aplicadas en otros ámbitos, como puede ser el clínico. Entre otros aspectos, se caracterizan por su complejidad (integradas por diversos componentes, interrelacionados entre sí y difícilmente delimitables ${ }^{8}$ ), por la vinculación de su efectividad al contexto en el que se aplican, y por presentar variables de resultado más complejas?

Actualmente, la evaluación de la efectividad de las intervenciones en el ámbito clínico se basa en el modelo propuesto por la MBE. Según este modelo, el método experimental y, más concretamente el ensayo clínico controlado y aleatorizado, es el diseño metodológicamente más robusto. En el ámbito de la salud pública las intervenciones frecuentemente no van a poder ser evaluadas mediante ensayos clínicos debido, entre otros motivos, a su alto coste, a motivos éticos o a que su puesta en práctica no es factible ${ }^{9,10}$. Todas estas circunstancias, además de la tradicional escasa inversión en investigación en salud pública, propician que el cuerpo de conocimiento existente sobre la efectividad de las intervenciones en salud pública generalmente se apoye en diseños metodológicamente menos robustos $y$, en consecuencia, catalogados con un menor "nivel de evidencia científica"

\section{LA SALUD PÚBLICA BASADA EN PRUEBAS}

En este contexto se propone el concepto de "Salud Pública Basada en la Evidencia"(SPBE), entendida por Jenicek como "el uso consciente, explícito y juicioso de la mejor evidencia en la toma de decisiones sobre la atención a comunidades y poblaciones en el campo de la protección de la salud, la prevención de la enfermedad y el mantenimiento y mejora de la salud" 11. Esta descripción subraya la necesidad de basar la toma de decisiones en salud pública en la mejor evidencia científica disponible e implica un proceso previo de recopilación y evaluación crítica de la literatura.

La SPBE se encuentra en la actualidad en un momento de reinterpretación del significado del término "evidencia científica" dentro del contexto de la salud pública. Son muchos los investigadores que advierten del peligro de trasladar sin matices los esquemas metodológicos planteados en la MBE al contexto de la salud pública porque así se asignaría el máximo nivel de evidencia únicamente a las intervenciones evaluadas mediante ensayos clínicos. No obstante, basar la evaluación de la efectividad de las intervenciones en salud pública exclusivamente en el ensayo clínico, además de presentar las limitaciones de índole práctico expuestas previamente, podría resultar inadecuado: este diseño a veces presenta una limitada validez externa (considera muestras de sujetos, ámbitos e intervenciones poco generalizables); desvincula la intervención de su contexto habitual de aplicación (eliminando, por tanto, la probable influencia del contexto en los resultados de la intervención) y no tiene en cuenta el proceso de implementación de la intervención ${ }^{12}$. En definitiva, las recomendaciones realizadas exclusivamente a partir de ensayos clínicos en ocasiones pueden llegar a ser poco útiles de cara a la acción y a la toma de decisiones en salud pública.

Por ello, entidades de reconocido prestigio dentro del mundo de la MBE ya apuestan claramente por el uso juicioso y explícito de otros diseños y metodologías, además del ensayo clínico, para la toma de decisio- 
nes. Proponen considerar todo el conocimiento relevante procedente de la investigación, planteamiento más próximo a la esencia de la MBE ("uso de las mejores pruebas procedentes de la investigación"). Un ejemplo representativo podría ser el del National Institute for Health and Clinical Excellence, NICE http://www.nice.org.uk/), que está desarrollando un método para establecer de una forma pragmática, sistemática y reproducible el nivel de evidencia de la efectividad de las intervenciones en salud pública y su correspondiente grado de recomendación. Este proyecto, todavía en su fase inicial, asigna el nivel de evidencia siguiendo el proceso habitual, es decir, basándose en el diseño y calidad de los estudios. Según este planteamiento, el ensayo clínico controlado y aleatorizado sigue constituyendo el diseño de elección para evaluar la efectividad de las intervenciones en salud pública (siempre que sea posible su realización). No obstante, para el establecimiento del grado de recomendación se pretende integrar la información procedente de otros tipos de diseños, como pueden ser los diseños observacionales, y de otros tipos de metodologías, como la investigación cualitativa. Estas formas alternativas de investigación pueden aportar información muy útil para juzgar la viabilidad y probabilidad de éxito de la intervención en el contexto local en el que se desea aplicar ${ }^{12}$.

Finalmente, para avanzar en la práctica de una SPBE se debería facilitar, entre otros, los siguientes aspectos: formación en el acceso y en el manejo de los recursos de información y en la valoración crítica de las evidencias disponibles ${ }^{13}$; incorporación de equipos profesionales multidisciplinares a esta nueva metodología de trabajo ${ }^{14}$; y, todo ello, sin olvidar el fomento del diálogo entre los investigadores y las instancias políticas. De este modo, estas últimas tendrían acceso a la información pertinente para decidir qué intervenciones deben ser aplicadas sobre el terreno o propuestas para futuras investigaciones.
El presente número de la revista incluye el excelente artículo de Morales Asencio y cols ${ }^{15}$ que describe estrategias para conocer con rigor los resultados de las intervenciones en salud pública. El artículo expone una clara visión de lo que implica el término SPBE en la actualidad y presenta la secuencia metodológica necesaria para practicar una salud pública basada en pruebas. Probablemente la lectura del artículo ayudará a aquellos profesionales que estén en fase de diseño o planificación de una intervención en salud pública ya que también describe un completo catálogo de recursos para localizar de una forma eficiente y exhaustiva pruebas útiles en la toma de decisiones. Adicionalmente, muchos de los recursos descritos presentan información actualizada y de calidad sobre la metodología empleada de forma específica en el campo de la SPBE.

Este artículo ofrece la oportunidad de plantearnos si las intervenciones en salud pública están siendo realmente evaluadas y, en caso afirmativo, hasta qué punto la metodología empleada en dicha evaluación es la más adecuada. En definitiva, la lectura del artículo debería suponer un estímulo para preguntarnos si la toma de decisiones en salud pública se basa actualmente en las mejores pruebas disponibles.

\section{BIBLIOGRAFÍA}

1. Guyatt, GH. Evidence-based medicine 1991. ACP Journal Club (A-16).

2. Sackett DL, Rosenberg WM, Gray JA, Haynes RB, Richardson WS. Evidence based medicine: what it is and what it isn't. BMJ 1996 13;312:71-72.

3. Muir Gray JA. Atención Sanitaria Basada en la evidencia. Cómo tomar decisiones en gestión y política sanitaria. Madrid: Churchill Livingstone España; 1997.

4. Acheson D. Report of the Committee of Inquiry into the future development of the Public Health functions and Community Medicine. London HMSO; 1988. 
5. Frenk J. The new public health. Annu Rev Public Health 1993;14:469-490.

6. Cid Ruzafa J, Rodríguez Artalejo F, Martín Moreno JM. ¿Hacia una Salud Pública Basada en la Evidencia?. Med Clin (Barc) 1999;112 Supl 1:106-110.

7. Nebot M. Evaluación en salud pública: ¿todo vale? [editorial]. Gac Sanit 2007;21:95-96.

8. Campbell M, Fitzpatrick R, Haines A, Kinmonth A, Sandercock P, Spiegelhalter D, et al. Framework for design and evaluation of complex interventions to improve health. BMJ 2000;321:694-696.

9. Victora CG, Habicht JP, Bryce J. Evidence-based public health: moving beyond randomized trials. Am J Public Health 2004;94:400-405.

10. Black N. Why we need observational studies to evaluate the effectiveness of health care. BMJ 1996;312:1215-18.
11. Jenicek M. Epidemiology, evidenced-based medicine, and evidence-based public health. J Epidemiol 1997; 7:187-97.

12. Health Development Agency. Grading evidence and recommendations for public health interventions: developing and piloting a framework [citado $7 \mathrm{de}$ febr 2008]. Disponible en: www.nice.org.uk/niceMedia/docs/grading_evidence.pdf.

13. Muir Gray JA. Evidence-based public healthwhat level of compliance is required? Journal of Public Health Medicine 1997;19:65-8.

14. Rodríguez Artalejo F. La salud pública basada en la evidencia [editorial]. Gac Sanit 1997;11:201-3.

15. Morales Asencio JM, Gonzalo Jiménez E, Martín Santos FJ, Morilla Herrera JC. Salud Pública basada en la evidencia. Recursos sobre la efectividad de intervenciones en la comunidad. Rev Esp Salud Pública. 2008; 81:5-20. 